01

\title{
Два связанных квазипериодических генератора, возбуждаемых гармоническим сигналом
}

\author{
(C) А.П. Кузнецов, ${ }^{1}$ Ю.В. Седова, ${ }^{1}$ Н.В. Станкевич1,2 \\ ${ }^{1}$ Саратовский фрилиал Института радиотехники и электроники им. В.А. Котельникова РАН, \\ 410019 Саратов, Россия \\ ${ }^{2}$ НИУ Высшая школа экономики, \\ 603155 Нижний Новгород, Россия \\ e-mail: sedovayv@yandex.ru
}

Поступило в Редакцию 17 мая 2021 г.

В окончательной редакции 7 июня 2021 г.

Принято к публикации 8 июня 2021 г.

Исследована возбуждаемая гармоническим сигналом система двух диссипативно связанных генераторов, способных демонстрировать автономные квазипериодические колебания. Представлены ляпуновские карты, выявляющие режимы инвариантных торов разной размерности и хаоса. Представлены фазовые портреты в стробоскопическом сечении и двойном сечении Пуанкаре. Обсуждено сосуществование различных режимов, в частности, бифуркации инвариантных торов.

Ключевые слова: квазипериодические колебания, связанные генераторы, ляпуновские карты, инвариантный тор, сечение Пуанкаре, квазипериодическая бифуркация.

DOI: 10.21883/JTF.2021.11.51519.145-21

\section{Введение}

Известно несколько примеров автономных генераторов, способных демонстрировать квазипериодические колебания. Это одна из схем Чуа [1], модифицированный генератор Анищенко-Астахова [2-7], семейство автономных генераторов с минимальной размерностью фазового пространства [8]. Квазипериодические колебания занимают своего рода промежуточное положение между периодическими и хаотическими колебаниями, однако динамика автономных систем с квазипериодичностью мало исследована. В то же время она представляет фундаментальный интерес. Два связанных квазипериодических генератора исследованы в работе [9]. Они демонстрируют достаточное количество интересных феноменов, включая эффект гибели колебаний, синхронную квазипериодичность идр. Представляет интерес исследование возбуждения такой системы внешним периодическим сигналом, т.е. изучение своего рода управления динамикой связанных квазипериодических генераторов. Как мы покажем, полученная картина является достаточно сложной, ее интересно сопоставить с задачей о возбуждении гармоническим сигналом двух связанных генераторов ван дер Поля $[10-12]$.

\section{1. Карты ляпуновских показателей}

В соответствии с [9] система связанных квазипериодических генераторов описывается уравнениями, в которые добавлено внешнее воздействие:

$$
\begin{gathered}
\ddot{x}_{1}-\left(\lambda_{1}+z_{1}+x_{1}^{2}-\beta x_{1}^{4}\right) \dot{x}_{1}+\omega_{0}^{2} x_{1} \\
+M_{c}\left(\dot{x}_{1}-\dot{x}_{2}\right)=a \cos \omega t, \\
\dot{z}_{1}=b\left(\varepsilon-z_{1}\right)-k \dot{x}_{1}^{2}, \\
\ddot{x}_{2}-\left(\lambda_{2}+z_{2}+x_{2}^{2}-\beta x_{2}^{4}\right) \dot{x}_{2}+\left(\omega_{0}+\Delta\right)^{2} x_{2} \\
+M_{c}\left(\dot{x}_{2}-\dot{x}_{1}\right)=0, \\
\dot{z}_{2}=b\left(\varepsilon-z_{2}\right)-k \dot{x}_{2}^{2},
\end{gathered}
$$

где $x_{1}, y_{1}=\dot{x}_{1}, z_{1}$ - переменные, характеризующие первый генератор, $x_{2}, y_{2}=\dot{x}_{2}, z_{2}-$ переменные второго генератора, $\Delta$ - частотная расстройка генераторов, $M_{C}$ - коэффициент диссипативной связи, $\omega$ - частота внешнего сигнала, $a$ - его амплитуда. Далее будем использовать значения параметров $b=1, \varepsilon=4, k=0.02$, $\lambda=-1, \beta=1 / 18, \omega_{0}=2 \pi$.

На рис. 1, $a$ показана ляпуновская карта системы (1) без воздействия (т.е. при $a=0$ ) на плоскости параметров частотная расстройка $\Delta-$ величина связи $M_{C}$ [9]. Различными цветами показаны типы режимов, цветовая палитра представлена рядом с рисунком. Визуализированы режимы гибели колебаний OD, синхронные периодические режимы Р, режим широкополосной квазипериодичности ВQ. На рис. 1 обозначения $T_{n}$ отвечают $n$ частотному тору. Отметим, что для потоковой системы один из ляпуновских показателей $\Lambda$ всегда равен нулю, и мы будем его отбрасывать. Таким образом, случай $\Lambda_{1}=0$ отвечает двухчастотному тору $T_{2}$. Увеличение размерности тора на единицу отвечает увеличению на единицу числа нулевых ляпуновских показателей. Так, 


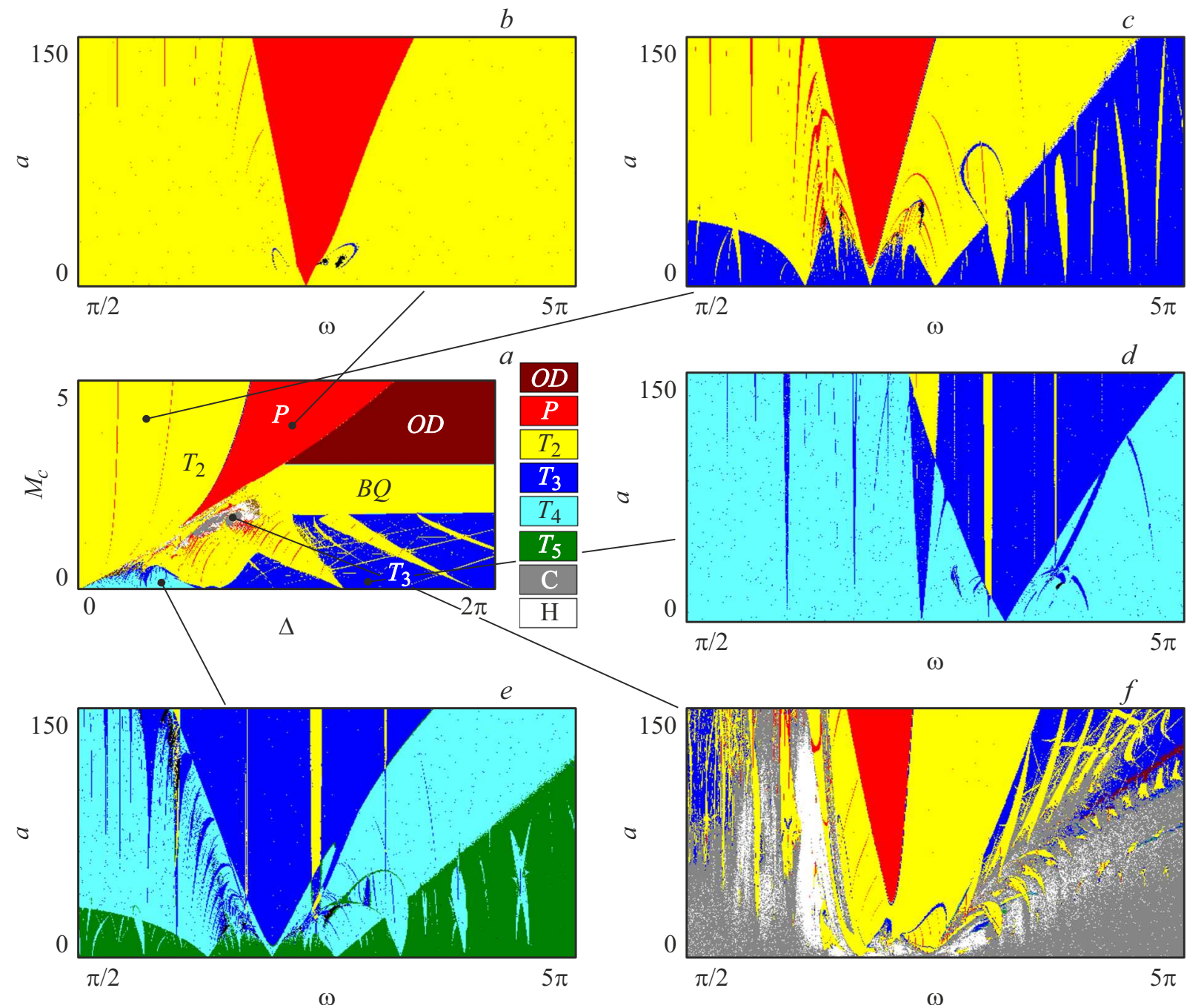

Рис. 1. Ляпуновские карты системы (1): $a-$ система без воздействия, $b-f-$ система с воздействием. Значения параметров: $b-\Delta=3.14, M_{c}=4 ; c-\Delta=0.9, M_{c}=4 ; d-\Delta=4.3, M_{c}=0.2 ; e-\Delta=0.75, M_{c}=0.05 ; f-\Delta=2.58, M_{c}=1.8$.

трехчастотному тору $T_{3}$ отвечает условие $\Lambda_{1}=\Lambda_{2}=0$ и т.д. Режимы хаоса $\mathrm{C}$ и гиперхаоса $\mathrm{CH}$ соответствуют одному и двум положительным показателям Ляпунова: $\Lambda_{1}>0$ и $\Lambda_{1}>, \Lambda_{2}>0$.

По периферии рис. 1 показаны карты возбуждаемой системы (1) на плоскости частота $\omega$ - амплитуда внешнего воздействия $a$ в выделенных точках автономной системы для некоторых характерных режимов: периодического режима $(b)$, двухчастотного тора $(c)$, трехчастотного тора $(d)$, четырехчастотного тора $(e)$ и хаотического режима $(f)$.

\section{2. Иллюстрации динамики и обсуждение результатов}

Рис. $1, b$ представляет случай периодического режима автономной модели. Как можно видеть, в возбуждаемой системе наблюдается периодический режим в виде языка Арнольда с острием на оси частот, погруженно- го в область двухчастотной квазипериодичности. Это традиционно для возбуждаемой модели с предельным циклом [13-15]. В то же время наблюдаются очень узкие языки синхронизации на субгармониках и малые области хаотической динамики.

Рис. 1, $c$ отвечает случаю двухчастотного тора $T_{2}$ в автономной системе. Значения параметров выбраны так, что в автономном режиме реализуется режим синхронной квазипериодичности, когда фазы генераторов взаимно захвачены, но система в целом демонстрирует квазипериодичность [9]. При малых значениях амплитуды воздействия $a$ на рис. 1, $c$ наблюдаются трехчастотные торы. В область их существования встроена система достаточно узких языков двухчастотных режимов, которые являются резонансными по отношению к трехчастотным торам. При больших значениях амплитуды воздействия возникают широкие области двухчастотных торов. Такая картина достаточно естественна - внешний сигнал захватывает одну из двух частот автономной модели. В то 

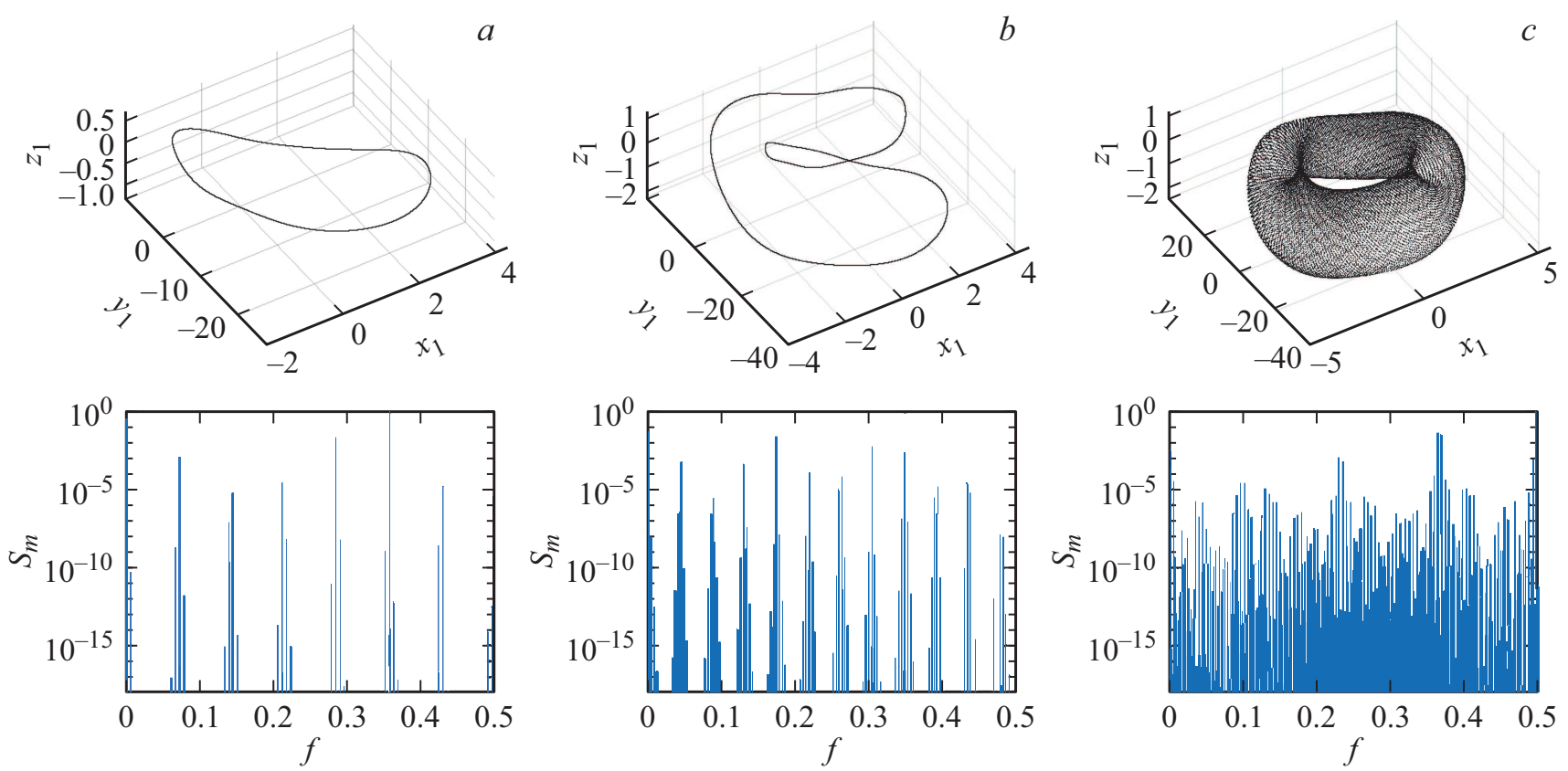

Рис. 2. Проекции фазовых портретов (верхний ряд) и соответствующие им спектры Фурье (нижний ряд) в различных точках карты $1 c ; f-$ нормированная частота, $S_{m}-$ спектральная интенсивность, выраженная в $\mathrm{dB}$. Значения параметров: $a-\omega=3.25 \pi$, $a=100 ; b-\omega=3.25 \pi, a=60 ; c-\omega=4.26 \pi, a=30$.

же время возникает достаточно обширная область чисто периодических режимов. Ее отличие от рис. $1, b-$ наличие порога по амплитуде воздействия. Таким образом, внешний сигнал в этом случае захватывает обе частоты автономной системы. Похожий эффект наблюдается и в системе двух связанных осцилляторов ван дер Поля [12]. Внешний сигнал при воздействии на связанные осцилляторы в режиме биений при достаточной амплитуде воздействия и определенной частотной расстройке захватывает не только непосредственно возбуждаемый осциллятор, но и второй (в этом плане интересно сопоставить рис. $1, c$ и рис. $1, c$ из [12]).

На рис. 2 показаны примеры характерных фазовых портретов в неавтономной системе (1) в стробоскопическом сечении (т.е. через период внешнего воздействия). Можно видеть замкнутую инвариантную кривую, отвечающую двухчастотному тору (рис. 2,a), удвоенный такой тор (рис. 2,b), а также трехчастотный тор (рис. 2,c). В нижнем ряду представлены фурье-спектры для указанных режимов. Стоит отметить обогащение спектров при возникновении трехчастотного тора.

На рис. 3 приведены графики трех старших показателей Ляпунова внутри одного из языков двухчастотных режимов вдоль линии $\omega=$ const $(a)$ и в ,Поперечном“ направлении $a=\mathrm{const}(b)$. В первом случае можно отметить бифуркацию удвоения двухчастотного тора DT. Ей отвечает точка, когда один из показателей $\Lambda_{1}=0$, а показатель $\Lambda_{2}$ остается отрицательным за исключением точки бифуркации, когда он оказывается нулевым. Во втором случае фиксируем бифуркацию двухчастотного тора SNT, когда величина показателя Ляпунова $\Lambda_{2}$ из нулевой становится отрицательной. Такая бифуркация является седло-узловой бифуркацией двухчастотного тоpa [16] с частичным захватом частот, и на поверхности трехчастотного тора рождается пара двухчастотных торов: устойчивый и седловой. На выходе из области двухчастотной квазипериодичности наблюдается слияние устойчивого и неустойчивого торов, что приводит к возникновению трехчастотного тора. Таким образом, боковыми границами языков двухчастотных торов на карте рис. $1, b$ являются линии седло-узловых бифуркаций торов.

На рис. $1, d$ и е представлены ляпуновские карты для случая трех- и четырехчастотного торов в автономной (без воздействия) системе. На рис. $1, d$ можно видеть язык трехчастотного тора, погруженный в область четырехчастотных торов. Боковыми границами языка являются линии седло-узловых бифуркаций трехчастотного тора. Внутри языка видны вертикальные окна двухчастотных торов, границами которых также являются седлоузловые бифуркации. Внутри области четырехчастотных торов наблюдаются узкие полосы трехчастотных торов, соответствующие языкам частичной синхронизации на высших гармониках.

На рис. 1,e в случае четырехчастотного тора в автономной системе при наличии внешнего воздействия язык трехчастотных торов сохраняется, но у него появляется небольшой порог по амплитуде воздействия. Он окружен областью четырехчастотной квазипериодичности, в которую встроено большое количество языков трехчастотных торов. В свою очередь, при малой амплитуде воздействия возникает область пятичастотных 

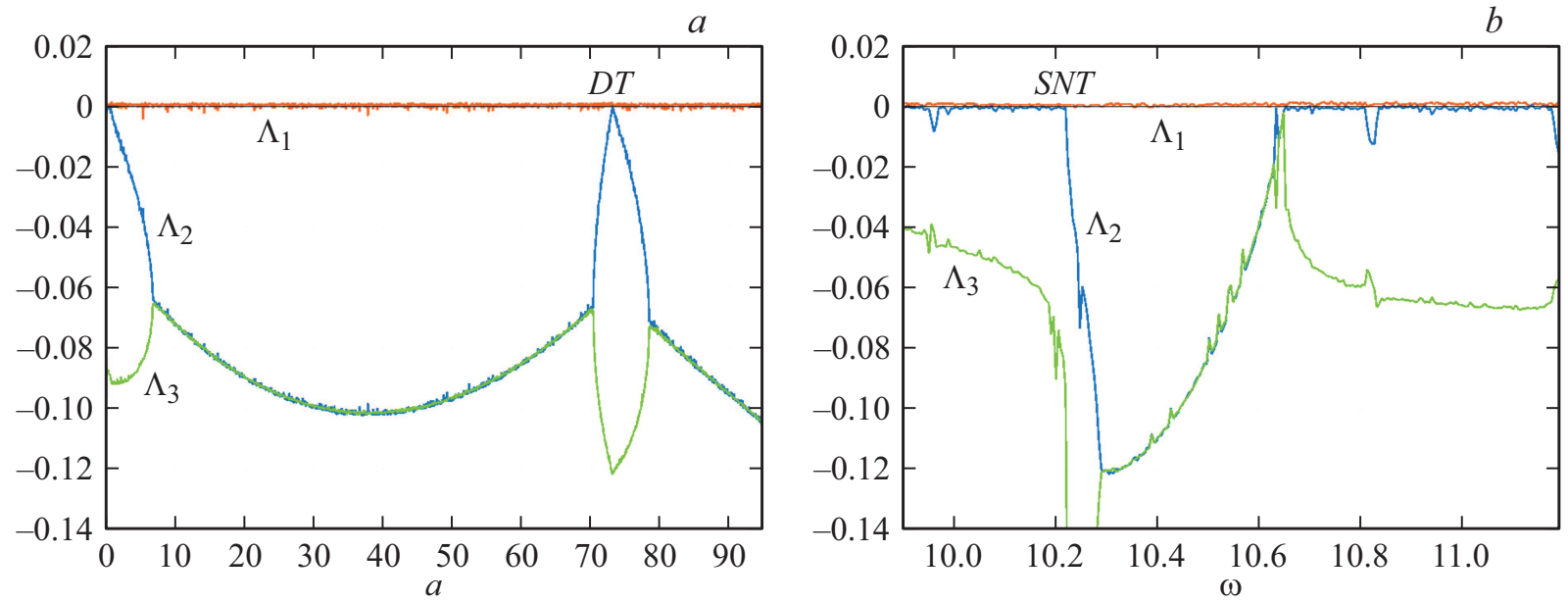

Рис. 3. Графики трех старших ляпуновских показателей системы (1) вдоль линий $\omega=10.47(a)$ и $a=23(b)$ на рис. $1, c$.
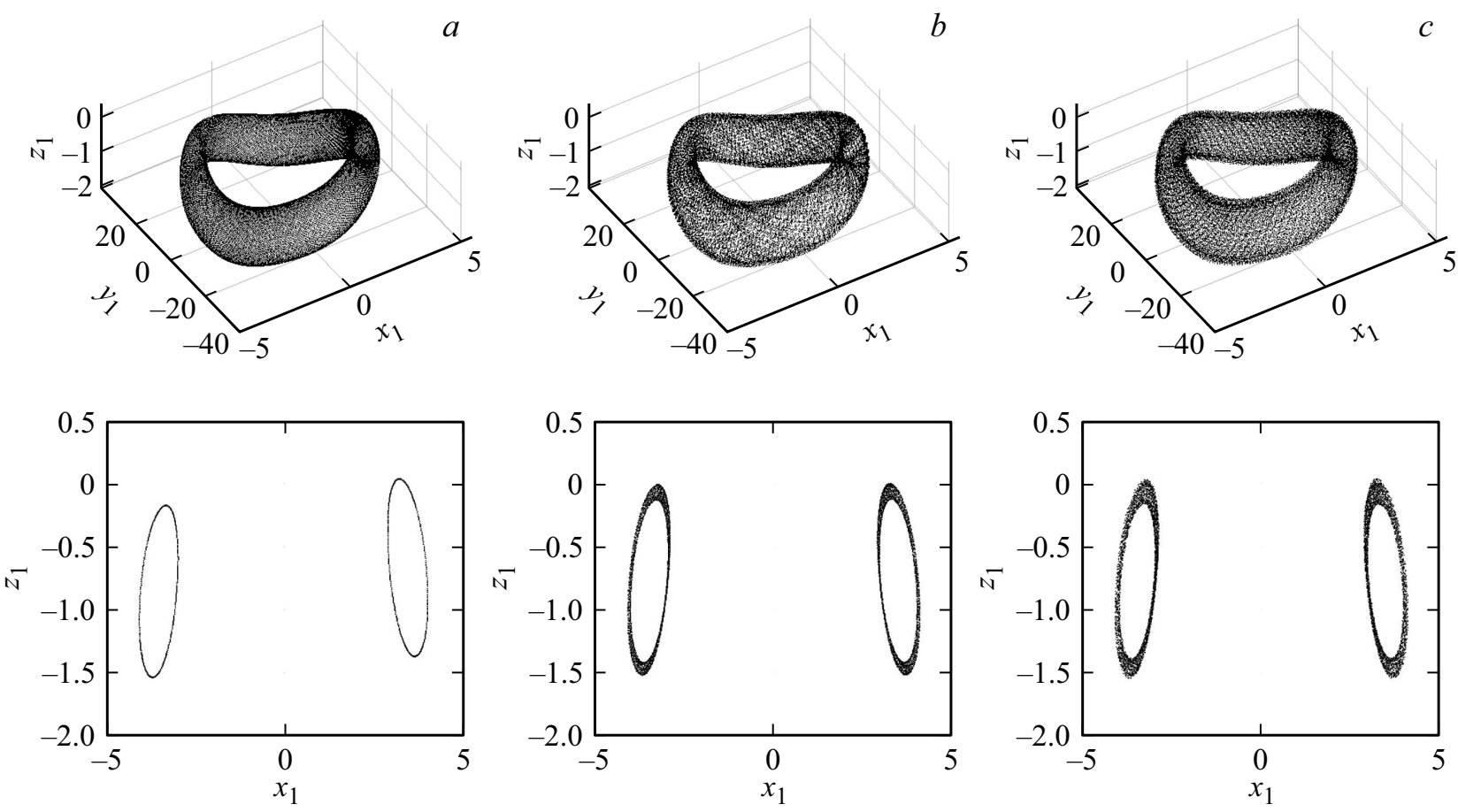

Рис. 4. Проекции фазовых портретов (верхний ряд) и двойные сечения Пуанкаре (нижний ряд) для трех-, четырех- и пятичастотных торов системы (1) для избранных точек карты, изображенной на рис. 1, e. Значения параметров: $a-\omega=2.25 \pi$, $a=80 ; b-\omega=4.15 \pi, a=110 ; c-\omega=4.75 \pi, a=30$.

торов, в которую встроены языки четырехчастотных торов, опирающиеся на ось частоты воздействия. Боковыми границами четырехчастотных торов являются седло-узловые бифуркации этих торов.

На рис. 4 в верхней строке приведены фазовые портреты трех-, четырех- и пятичастотных торов для избранных точек карты рис. 1, е. Подчеркнем, что визуально они практически неотличимы. Для выделения трехчастотных торов следует построить двойное сечение Пуанкаре. Отметим, что обычное сечение Пуанкаре для системы, находящейся под внешним гармоническим воздействием, представляет собой набор дискретных точек, получаемых в результате стробоскопического сечения. Для построения двойного сечения необходимо учитывать только те точки из упомянутого дискретного набора, которые попадают в некоторый тонкий слой фазового пространства, например, в данном случае использовалось условие $\left|y_{1}\right| \leq 0.01$. Результат двойного сечения (стробоскопического сечения и сечения плоскостью $\left.y_{1}=0\right)$ фазового пространства системы (1) представлен на рис. 4 в нижней строке. Двойное сечение трехчастотного тора представляет собой два замкнутых овала в отличие от четырех- и пятичастотных торов (для различения последних двойные сечения Пуанкаре 

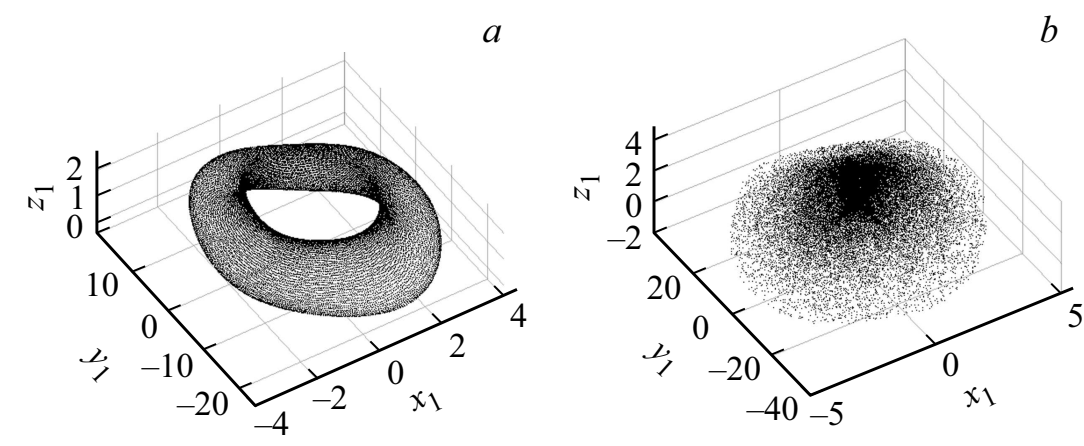

$b$
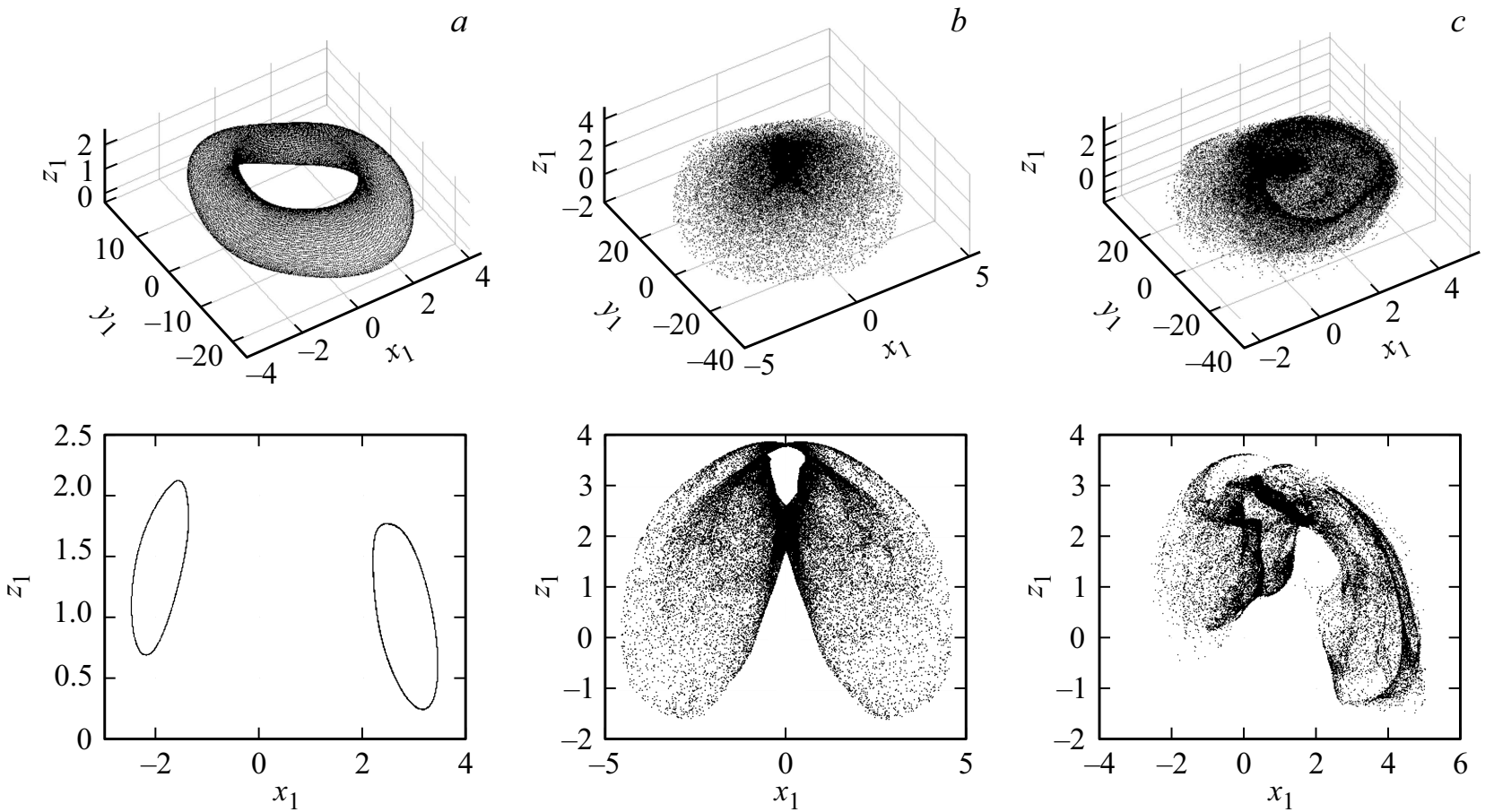

Рис. 5. Проекции фазовых портретов в различных точках карты рис. $1, f$ (верхний ряд) и соответствующие им двойные сечения Пуанкаре (нижний ряд). Значения параметров: $a-\omega=3.75 \pi, a=150 ; b-\omega=4.5 \pi, a=20 ; c-\omega=1.6 \pi, a=110$.

неинформативны и целесообразно применить тройное сечение, например, используя дополнительную секущую плоскость $\left.z_{1}=-1\right)$.

Наконец, на рис. $1, f$ показана ляпуновская карта для случая хаотической динамики системы без воздействия. В этом случае для малых амплитуд воздействия, как и следовало ожидать, наблюдается хаотический режим. Однако с ростом амплитуды воздействия наблюдается аналогичный [13] эффект подавления хаоса внешним сигналом с возникновением периодического (синхронного) режима. Особенность рассматриваемой системы состоит в том, что подавление хаоса приводит не только к периодическому режиму, но и к режимам двух- и трехчастотных торов. Этот факт иллюстрирует рис. 5, на котором показан фазовый портрет и двойное сечение Пуанкаре для трехчастотного режима $(a)$. Также с увеличением амплитуды сигнала можно наблюдать развитие хаотической динамики, формирование так называемого гиперхаотического режима, который характеризуется двумя положительными показателями Ляпунова в спектре. Иллюстрации хаотического и гиперхаотического режимов представлены на рис. $5, b$ и $c$ соответственно. Таким образом, с помощью внешнего сигнала можно управлять хаотическим поведением в системе двух связанных генераторов, причем как стабилизировать хаос, так и усложнить развитием гиперхаоса.

На рис. $1, f$ можно видеть, что в область трехчастотных торов встроены языки двухчастотных торов (верхняя правая часть карты, справа от основного языка двухчастотных торов). Эти языки, однако, „переверну- тые“, они расширяются при уменьшении амплитуды воздействия и начинают перекрываться с возникновением хаоса. Отметим, что в этом случае в основном наблюдается хаос с одним положительным показателем Ляпунова, что характерно для хаоса, возникшего в результате потери гладкости инвариантной кривой. Гиперхаос преимущественно развивается слева от основного языка двухчастотной квазипериодичности.

\section{Заключение}

Таким образом, система возбуждаемых связанных квазипериодических генераторов демонстрирует богатое разнообразие режимов на основе инвариантных торов разной размерности. В основном наблюдается система языков инвариантных торов, погруженных в область торов более высокой размерности. Границами языков являются седло-узловые бифуркации торов соответствующей размерности. Внутри языков наблюдаются бифуркации удвоения торов. Внешний сигнал может инициировать квазипериодичность разной размерности в случае хаотической динамики автономной системы. Возможно управление хаосом: его стабилизация или развитие в гиперхаотический режим.

\section{Финансирование работы}

Исследование выполнено за счет гранта Российского научного фонда № 21-12-00121, https://rscf.ru/project/21$12-00121 /$. 


\section{Конфликт интересов}

Авторы заявляют, что у них нет конфликта интересов.

\section{Список литературы}

[1] T. Matsumoto. Proceed. IEEE, 75(8), 1033 (1987). DOI: $10.1109 /$ PROC. 1987.13848

[2] В.С. Анищенко, С.М. Николаев. Письма в ЖТФ, 31 (19), 88 (2005). [V. Anishchenko, S. Nikolaev. Tech. Phys. Lett., 31 (10), 853 (2005). DOI: 10.1134/1.2121837]

[3] В.С. Анищенко, С.М. Николаев. Нелинейная динамика, 2 (3), 267 (2006). DOI: 10.20537/nd0603001

[4] V. Anishchenko, S. Nikolaev, J. Kurths. Phys. Rev. E, 73, 056202 (2006). DOI: 10.1103/PhysRevE.73.056202

[5] V. Anishchenko, S. Nikolaev, J. Kurths. Phys. Rev. E, 76, 046216 (2007). DOI: 10.1103/PhysRevE.76.046216

[6] V.S. Anishchenko, S. M. Nikolaev. Intern. J. Bifurcation and Chaos, 18 (09), 2733 (2008). DOI: $10.1142 / \mathrm{S} 0218127408021956$

[7] В.С. Анищенко, В.В. Астахов, Т.Е. Вадивасова. Регулярные и хаотические автоколебания. Синхронизация и влияние флуктуаций. Учебник-монография (Издат. Дом „Интеллект“, Долгопрудный, 2009)

[8] A.P. Kuznetsov, S.P. Kuznetsov, E. Mosekilde, N.V. Stankevich. The Europ. Phys. J. Special Topics, 222, 2391 (2013). DOI: $10.1140 /$ epjst/e2013-02023-x

[9] A.P. Kuznetsov, S.P. Kuznetsov, N.A. Shchegoleva, N.V. Stankevich. Physica D, 398, 1 (2019). DOI: 10.1016/j.physd.2019.05.014

[10] V. Anishchenko, S. Astakhov, T. Vadivasova. Europhys. Lett., 86 (3), 30003 (2009). DOI: 10.1209/0295-5075/86/30003

[11] В.С. Анищенко, С.В. Астахов, Т.Е. Вадивасова, А.В. Феоктистов. Нелинейная динамика, 5 (2), 237 (2009). DOI: $10.20537 / \mathrm{nd} 0902006$

[12] А.П. Кузнецов, И.Р. Сатаев, Л.В. Тюрюкина. Письма в ЖТФ, 36 (10), 73 (2010).

[13] A. Pikovsky, M. Rosenblum, J. Kurths. Synchronization: a Universal Concept in Nonlinear Sciences (University Press, Cambridge, 2003)

[14] В.С. Анищенко. Сложные колебания в простых системах (Наука, М., 1990)

[15] П.С. Ланда. Автоколебания в системах с конечным числом степеней свободы (Наука, М., 1980)

[16] R. Vitolo, H. Broer, C. Simó. Regular and Chaotic Dynamics, 16, 154 (2011). DOI: 10.1134/S1560354711010060 